\title{
Is the venoarterial extracorporeal membrane oxygenation circuit your frenemy?
}

\author{
David L. Joyce, MD
}

\footnotetext{
From the Division of Cardiothoracic Surgery, Department of Surgery, Froedtert and the Medical College of Wisconsin, Milwaukee, Wis.

Disclosures: Author has nothing to disclose with regard to commercial support.

Received for publication Sept 27, 2017; accepted for publication Oct 6, 2017; available ahead of print Nov 7 , 2017.

Address for reprints: David L. Joyce, MD, Division of Cardiothoracic Surgery, Department of Surgery, Froedtert and the Medical College of Wisconsin, 9200 W Wisconsin Ave, Milwaukee, WI 53226 (E-mail: djoyce@mcw. edu).

J Thorac Cardiovasc Surg 2018;155:641-2

$0022-5223 / \$ 36.00$

Copyright (c) 2017 by The American Association for Thoracic Surgery

https://doi.org/10.1016/j.jtcvs.2017.10.010
}

When facing a dramatic crisis in the operating room, most surgeons would do well to abide by Norm Shumway's second law: "The pump is your friend." 1 The hemodynamic stability achieved with extracorporeal support has been exploited by three generations of surgeons and continues to provide a fail-safe strategy when things go awry. Despite its numerous advantages, however, cardiopulmonary bypass (CPB) comes at a steep pricethe need for full anticoagulation. Venoarterial extracorporeal membrane oxygenation (VA ECMO) has gained favor as a short-term bridging strategy for patients requiring cardiopulmonary support in the days after surgery. As with $\mathrm{CPB}$, however, the need for anticoagulation generally results in a reluctance to add coagulopathy to the list of ongoing challenges that typically accompany this type of patient. In their article in this issue of the Journal, "Short-term Venoarterial Extracorporeal Membrane Oxygenation for Massive Endobronchial Hemorrhage After Pulmonary Endarterectomy," Guth and colleagues ${ }^{2}$ describe an ingenious method for leveraging all the advantages of VA ECMO support before the consequences of anticoagulation and pulmonary stasis become relevant.

Pulmonary endarterectomy can cure chronic thromboembolic pulmonary hypertension, but, as a technically demanding procedure that is infrequently performed in most centers, it carries several unique risks-particularly the potential for massive endobronchial hemorrhage. For patients with this complication, survival depends on the immediate execution of a proven rescue strategy. These maneuvers, nicely described by the San Diego group ${ }^{3}$ and others, ${ }^{4,5}$ should be mentally rehearsed by the surgeon before the start of each endarterectomy. Although only a few patients in the series of Guth and colleagues ${ }^{2}$ had this complication develop (as expected in such a highly experienced group), this ultra-short term use of VA ECMO yielded results that justify its inclusion in the commonly accepted emergency protocols. The critical importance of anticipating and identifying massive

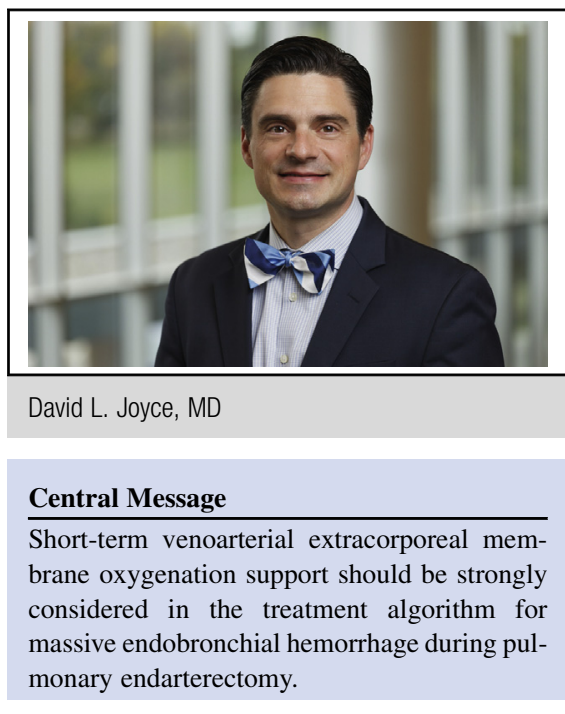

See Article page 643.

endobronchial hemorrhage and then managing it with VA ECMO is illustrated in the modified Jamieson flow diagram (Figure 1$)^{3}$ and is consistent with the requirements outlined in the study of Guth and colleagues. ${ }^{2}$

Beyond their practical relevance in the setting of complicated pulmonary endarterectomies, Guth and colleagues'

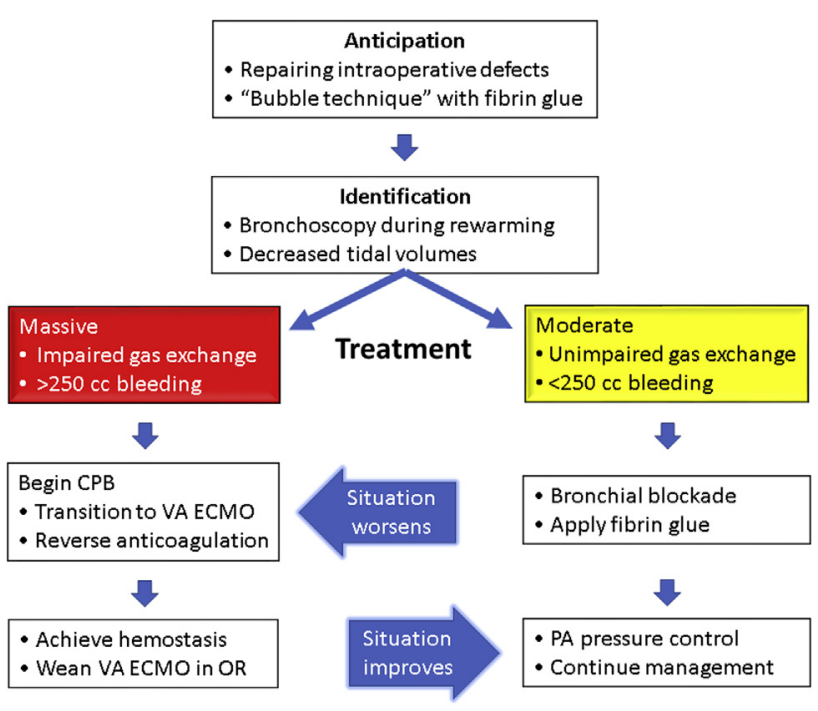

FIGURE 1. This flow diagram represents a modification of the traditional approach $^{2}$ to endobronchial hemorrhage during pulmonary endarterectomy. $C P B$, Cardiopulmonary bypass; VA ECMO, venoarterial extracorporeal membrane oxygenation; $P A$, pulmonary artery; $O R$, operating room. 
findings ${ }^{2}$ could be a catalyst for generating hypotheses about managing related intraoperative crises. The combination of coagulopathy and marginal right-ventricular function occurring after prolonged CPB is all too familiar to transplant surgeons and those implanting mechanical circulatory support devices. Likewise, many aortic surgeons will appreciate the occasional need for enhanced hemodynamic support after a complex circulatory arrest procedure when trying to restore hemostasis with "packing, protamine, products, and patience." Whether a brief period of extracorporeal support with a heparin-coated circuit offers a friendly alternative during these and related encounters, however, is a question that, in the words of W. W. Mayo, must be "left open for further thought and research." 6

\section{References}

1. Robbins RC. Norman E. Shumway. Clin Cardiol. 2000;23:462-6.

2. Guth S, Wiedenroth CB, Wollenschläger M, Richter MJ, Ghofrani HA, Arlt M, et al. Short-term venoarterial extracorporeal membrane oxygenation for massive endobronchial hemorrhage after pulmonary endarterectomy. J Thorac Cardiovasc Surg. 2018;155:643-9.

3. Manecke GR Jr, Kotzur A, Atkins G, Fedullo PF, Auger WR, Kapelanski DP, et al. Massive pulmonary hemorrhage after pulmonary thromboendarterectomy. Anesth Analg. 2004;99:672-5.

4. Shetty DP, Nair HC, Shetty V, Punnen J. A novel treatment for pulmonary hemorrhage during thromboendarterectomy surgery. Ann Thorac Surg. 2015;99: e77-8.

5. Reddy S, Rajanbabu BB, Kumar NK, Rajani I. Temporary clamping of branch pulmonary artery for pulmonary hemorrhage after endarterectomy. Ann Thorac Surg. 2013;96:1459-61.

6. Olsen L, Saunders RS, McGinnis JM. Patients Charting the Course: Citizen Engagement and the Learning Health System: Workshop Summary. Washington, DC: National Academies Press; 2001:53. 